\title{
Engine knock margin control using in-cylinder pressure data: preliminary results
}

\author{
Giulio Panzani ${ }^{1}$, Olga Galluppi ${ }^{1}$, Donald Selmanaj ${ }^{2}$, Sergio Savaresi ${ }^{1}$, \\ Jonatan Rösgren ${ }^{3}$ and Christopher H. Onder ${ }^{4}$
}

\begin{abstract}
Knock is an undesired phenomenon occurring in spark ignited engines and is controlled acting on the spark timing. This paper presents a closed-loop architecture that makes possible to address the knock control problem with a standard model-based design approach. An engine knock margin estimate is feedback controlled through a PI regulator and its target value is computed starting from the desired knock probability. A black-box modelling approach is used to identify the dynamics between the spark timing and the knock margin and a traditional model-based controller synthesis is performed. Experimental results at the test bench show that, compared to a conventional strategy, the proposed approach allows for a better compromise between the controller speed and the variability of the spark timing. Moreover, another advantage w.r.t. the conventional strategies is that closed-loop performance prove to be constant for different reference probabilities, leading to a more regular engine behaviour.
\end{abstract}

\section{INTRODUCTION}

Knock is a limiting phenomenon in spark ignited engines; it is the autoignition of the end gas in the combustion chamber which generates large pressure oscillations causing potential engine damages and performance decreases, [1]. However, in order to maximize the engine efficiency and in particular during low speed and high torque operations, the engine needs to run close to the knocking condition: thus a closed-loop knock margin regulation, acting on the spark timing, is a crucial component.

The scientific literature has dedicated considerable effort to the problem of knock sensing and knock control. While the detection of knock events is a relatively easy task [2]-[5], the control part is more challenging. Due to the stochastic behaviour of engine knock and its binary nature (i.e an engine cycle knocks or not), the use of classical standard control strategies is limited and not-trivial. Strategies proposed in literature can be divided in two groups: those controlling properties of knock, derived by knock occurrence and others controlling a knock-related (usually model-based) metrics.

Among the strategies controlling the stochastic properties of knock events, the most simple one is referred to in

This work was supported by the European Unions Horizon 2020 Research and Innovation Programme under grant 634135.

1 Dipartimento di Elettronica, Informazione e Bioingegneria, Politecnico di Milano, Milano 20133, Italy. Corresponding author: giulio.panzani@polimi.it

2 Department of Automation, Polytechnic University of Tirana, Tirana 1001, Albania.

3 Wärtsilä Finland Oy, Vaasa 65101, Finland.

${ }^{4}$ Institute of Dynamic Systems and Control, Department of Mechanical and Process Engineering, Swiss Federal Institute of Technology, Zürich 8092, Switzerland. literature as the conventional strategy. It consists of rapidly retarding the spark timing if a knock event is observed, and slowly advancing the timing during non-knocking cycles [6]. Due to its easy implementation and tuning, the conventional knock controller is widely used in industrial applications. However, it results in a late average (i.e., low efficiency) and a high variance of the spark timing. More advanced methods monitor the cumulative summation of knock events and compare it with the desired knock rate [7], [8]. Instead of acting at each knock event, those controllers retard the spark timing when the difference between the observed and the desired knock rate exceeds a positive threshold and advances the spark timing when the difference falls below a negative threshold. The methods proposed in [7] and [8] use fixed amplitudes of the retarding and the advancing actions. A further improvement can be made by relating the action intensity to the discrepancy between the observed and the desired knock rate: the likelihood ratio is an indicator of this discrepancy and is employed in [9]. The so-called likelihood-based approach shows satisfactory results on both simulation end experimental data [10]. Although effective, advanced stochastic knock controllers rely on non-standard tuning procedures, have a delayed transitory response and present a sub-optimal trade-off between the controller speed and the steady-state variability of the spark timing.

Due to the difficulty of modelling the combustion inside a cylinder chamber, methods based on the control of a knockrelated metrics are less developed. The most trivial knock metric is the cycle peak pressure [11]. Cycles with higher peak pressures are more likely to be knocking, thus the maximum pressure can be controlled at a reference value that is a compromise between the engine torque output and the knock tendency. Another possibility consists in quantifying the knock intensity through the engine casing acceleration to build a knock energy indicator controlled via a proportional integral (PI) controller [12]. However, the estimation of the mean and the variance of the energy indicator slows the controller action. An alternative method makes use of an Arrhenius-like function to model the autoignition of the endgas and the probability of knock, [13].

The knock control strategy proposed in this paper is based on the estimation approach introduced in [14], where the authors build a gray-box model of the knock margin that proves to effectively extract important information from the cylinder pressure traces and describes the knock behaviour in various engine operating conditions, outperforming more traditional physics-based approaches. The estimator provides, 
for each engine cycle, the knock margin (i.e. the distance from knocking conditions) and an estimate of the expected knock probability.

In the present work a closed-loop control is used to regulate the estimated engine knock margin at the target value, which is computed starting from the desired knock rate. In addition to the use of the real time estimated knock margin as feedback variable, another significant contribution is that the tuning of the proposed controller makes use of classical black-box identification and model-based control design techniques, overstepping the difficulties of the aforementioned approaches.

The experimental results at the test bench show the effectiveness of the method, in addition to the repeatability and satisfactory closed-loop performance. A comparison with a conventional knock control strategy is also provided.

The paper is organized as follows. Section II recalls the knock margin estimation and describes the black-box modelling approach, which is at the basis of the controller design. Section III introduces the proposed control scheme and describes the conventional controller used for the comparison. In Section IV the experimental results are shown and discussed. The paper ends with some concluding remarks and future works.

\section{KNOCK MARGIN MODELLING AND BLACK-BOX IDENTIFICATION}

\section{A. Experimental setup}

The experimental test bench is equipped with an electric brake and a four-stroke SI engine installed in ETH facilities in Zürich. The engine is equipped with direct gasoline injection, a turbocharger, and a variable valve camshaft. Injection timing was set at $270^{\circ}$ before top dead center (bTDC) and intake valve closing was set at $180^{\circ}$ bTDC. Table I summarizes the main characteristics of the engine. A sensor at the exhaust measures the air-to-fuel ratio which

TABLE I

MAIN ENGINE CHARACTERISTICS

\begin{tabular}{lcc} 
& Units & Value \\
\hline Cylinders & {$[-]$} & 3 \\
Combustion type & {$[-]$} & SI \\
Unitary displacement & {$[\mathrm{cc}]$} & 499.6 \\
Bore & {$[\mathrm{mm}]$} & 82 \\
Compression ratio & {$[-]$} & $10.1: 1$
\end{tabular}

is regulated at stoichiometric conditions by closed-loop using the amount of fuel injected. The air mass flow is measured by a hot-film anemometer and controlled by a waste-gate valve at the turbocharger. All the tests are performed at a speed of $1500 \mathrm{rpm}$, an air mass flow of $667 \mathrm{mg} / \mathrm{stroke}$, a coolant temperature of $85^{\circ} \mathrm{C}$, a rail pressure of 200 bar, and throttle fully open. Time units are considered in crank angle domain (one time unit is a complete crank angle cycle). Shown data only refers to cylinder 1 , as other cylinders response appears similar.

\section{B. System modelling and identification}

Knock margin is not a directly available quantity and it has to be estimated using available engine measures, in particular the in-cylinder pressure ones. Moreover, in order to set the control problem in a standard model-based design framework, a dynamic model between the spark timing (ST) and the estimated knock margin is identified.

A block scheme diagram of the system under analysis is shown in Figure 1. The solid line block represents the physical system i.e., the engine itself, that is considered as a SISO system whose input is the control variable (ST) and the output is the measured in-cylinder pressure $p_{c y l}$. The control results shown in this paper refer to a single engine operating point, thus no additional signals are considered, whereas of course the relationship between the spark timing and the incylinder pressure highly depends on other parameters like engine speed, intake air temperature etc.

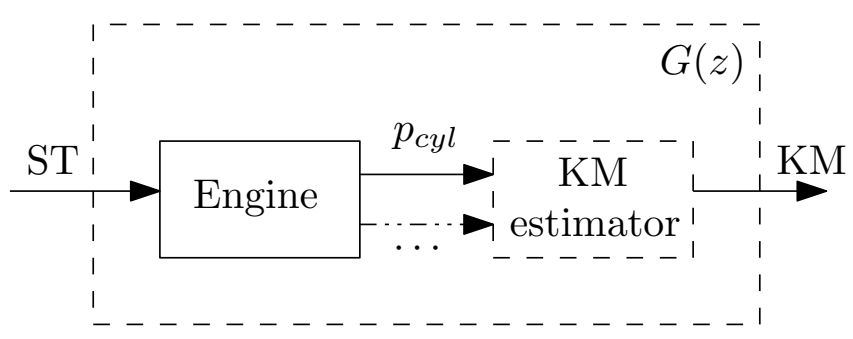

Fig. 1. Block diagram representation of the open loop system.

The dashed blocks, represent two additional non-physical systems that are required to properly set the hereby proposed knock control strategy. The knock margin estimation block is based on the approach presented in [14] which is here briefly summarized. The estimation procedure, which uses the incylinder pressure measure, is made up of two steps: the first exploits a Principal Component Analysis (PCA) to extract relevant common features (the eigenpressures) from the pressure traces, in order to reduce the algorithm complexity and to condense relevant information within few variables $\left(\gamma_{i}, i=1, \ldots, n\right.$, where $n$ depends on the desired model accuracy). In a second step, a logistic regression model is trained: this model combines the pressure features in a proper linear model (whose coefficients are $\beta_{i}, i=1, \ldots, n$ and $\alpha$ ) and relates them to the measured knock probability, which is indirectly estimated by using knocking/not-knocking cycles data.

As a result, the found linear combination defines the knock margin:

$$
K M=\sum_{i}^{n} \beta_{i} \gamma_{i}+\alpha
$$

which can be used to estimate the knock probability using the logit function:

$$
P_{\mathrm{est}}=\frac{e^{K M}}{1+e^{K M}}
$$

For more details the reader is referred to [14]. A stepwise ST test, ranging from 0 to $13^{\circ}$ bTDC, has been used 
for model identification. Considering that a single engine operating point is here analysed, it has been found that a single feature $\gamma_{1}$ is enough to properly model engine knock probability. However it should be recalled that the knock margin estimation method provides excellent results even in a wider operating point scenario, for varying engine speed, load, intake temperatures and air-to-fuel ratios at the cost of increasing the number of features considered and the additional engine operating point descriptive measures. Therefore, the single operating point simplification here considered should not be seen as a limitation of the proposed knock control strategy. The knock probability modelling results are shown in Figure 3.

The outer block named $G(z)$ completes the overall system; it is meant to describe the whole system dynamics between the control variable and the estimated knock margin and as such it will be used for the design of the knock margin controller, within a standard SISO model-based framework. A comment on the proposed approach is important: another possible choice would have been to identify the engine dynamics, from ST to the in-cylinder pressure, and then to compute the overall model as a series connection between such model and the knock margin estimator. However the engine pressure modelling, though undertaken with a black-

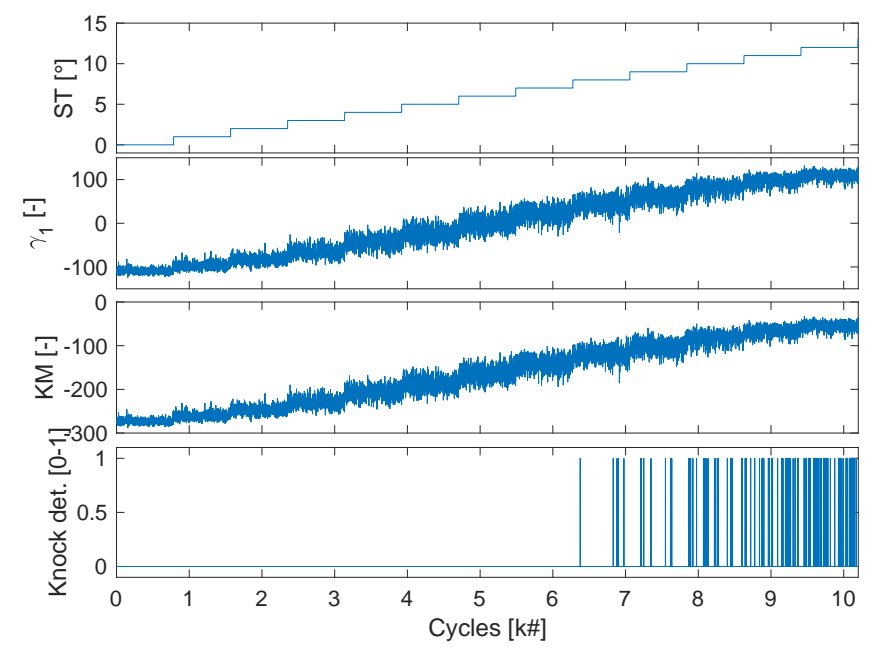

Fig. 2. Spark timing stepwise test example for model identification.

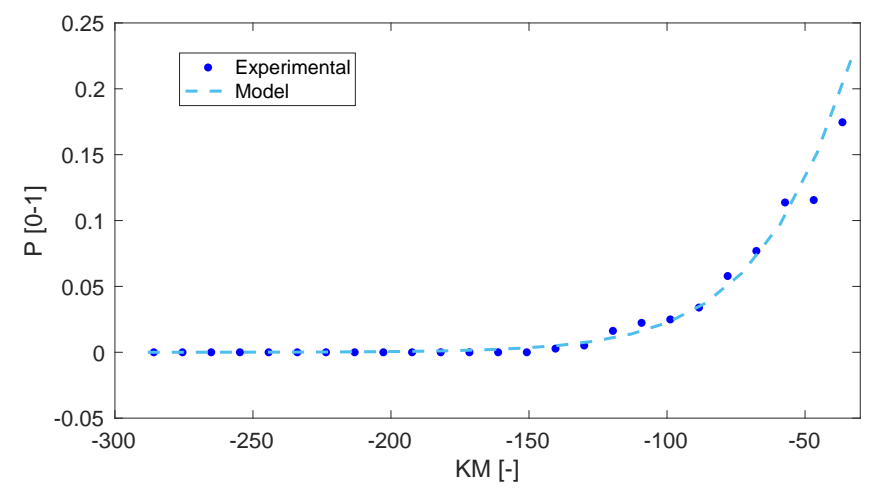

Fig. 3. Knock probability modelling results. box approach, is well known to be a difficult task and usually requires nonlinear complex models; aiming at the knock margin control, a precise description is not required since the knock margin estimation steps already extract from incylinder pressure data all the necessary information (thanks to the PCA) combining them in the appropriate nonlinear fashion (thanks to the logit function).

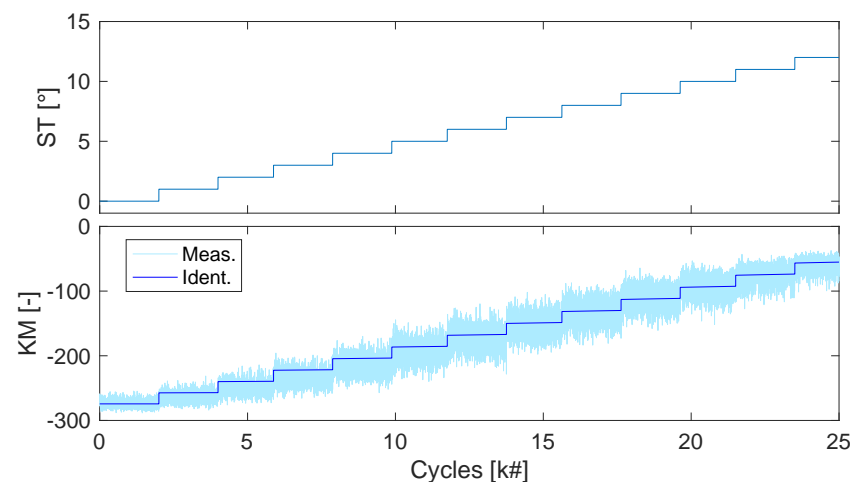

Fig. 4. ARMAX identification of $G(z)$.

In Figure 4 identification results are shown: the identified model output is compared to the measured one on the ST step test (using validation data). The identified model is a linear first order ARMAX model and turns out to have a negligible dynamic (the step response steady-state is reached after 2 cycles). From this comparison it is possible to appreciate how even a simple linear model is capable of describing the relationship between the spark timing and the knock margin despite the wide range of ST analysed. Moreover, the wellknown highly stochastic nature of knock, which cannot be captured by the model, is visible.

\section{KNOCK MARGIN CONTROL ARCHITECTURE}

The proposed control architecture is shown in Fig. 5. The knock margin, expressing the distance from the knocking condition, is provided by the knock estimator which uses cylinder pressures. The margin is used as a virtual measurement and is closed-loop controlled acting on the spark timing. The PI controller is tuned on the ARMAX model that relates the spark timing (i.e., control variable) and the knock margin (i.e., output variable). The highly stochastic nature of knock, which is visible in the modelling results, causes added noise on the estimated knock margin. The PI controller should be tuned to cut-off the high frequency noise while reacting to slow variations of the margin. As expected a slower controller presents slower responses to changes in the reference and lower variance of the control variable. In addition to the knock margin, the logistic regression modelling approach provides an open-loop estimate of the knock probability. In fact, equation (1) shows the relation between the knock margin and the estimated probability. Given the desired knock rate, this expression can be inverted to obtain a reference for the knock margin controller.

The knock margin control approach is compared with the 


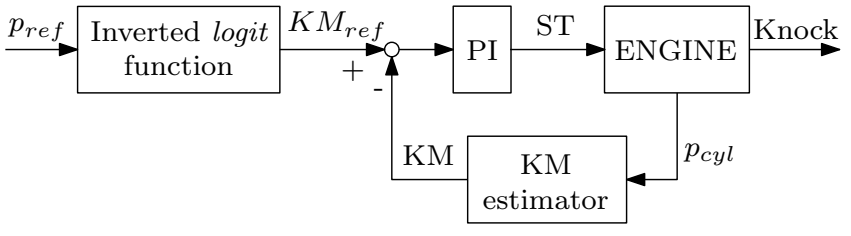

Fig. 5. Knock margin control scheme.

well-known conventional controller. Equation (2),

$$
S T(j)= \begin{cases}S T(j-1)-K_{\text {ret }} & \text { if knock, } \\ S T(j-1)+K_{a d v} & \text { otherwise, }\end{cases}
$$

implements the strategy where $S T(j)$ is the spark timing at cycle count $j, K_{r e t}$ is the retarding quantity on knock events, and $K_{a d v}$ is the advancing quantity during non-knocking cycles. Under the assumption of stable operation (i.e., knock occurs deterministically at a fixed spark timing), advances and retards cancel each other, and the following equation relates the desired knock probability $\left(P_{r e f}\right)$ to the controller parameters (see [15]):

$$
K_{a d v}=\frac{P_{r e f}}{1-P_{r e f}} K_{r e t} .
$$

While $P_{r e f}$ is an engine design parameter related to its structural strength, the variable $K_{\text {ret }}$ can be considered as a control parameter and determines the reactivity of the controller. Large values of $K_{\text {ret }}$ allow for shorter controller transients, but increase the variance and retard the average of the spark timing at steady-state operation. Here, the amplitude of $K_{r e t}$ is set to $1.5^{\circ}$, which is typical for production systems and provides a good trade-off between speed and spark timing variability.

\section{Closed-LOOP EXPERIMENTAL RESUltS}

\section{A. Knock margin controller experimental validation}

Knock margin control is tested using time-varying profiles of demanded knock probability $P_{r e f}$ and its correspondent $K M$ reference. Step responses have been selected in order to evaluate controller performance during transients as well as its steady-state behaviour. In particular, the step duration was set to 3500 cycles in order to have enough data to correctly compute steady-state performance. In the following, the estimated knock probability has been evaluated using a moving average (MA) filter with a window of 1000 samples giving an accuracy in the estimation of $0.1 \%$.

In Figure 6 the functioning of the knock margin closedloop control is shown, for the slowest controller tuning. In the first plot the control variable is shown and in the second the controlled variable along with the reference value. The third plot illustrates the comparison between probability predicted by (1) and with the one estimated with the moving average filter. It should be remarked that the transient behaviour of the estimated probability is due to the MA filter dynamics that requires 1000 cycles to get to steady-state; it is thus reasonable to consider its estimate reliable after half of the step duration. In the last plot the detected knock events are shown.

The experimental results validate the expected and desired behaviour of the proposed knock control approach. Reference knock margin is tracked as requested, meeting the desired knock probability target and repeatability of the performance can be appreciated by noting the similar closed-loop behaviour of subsequent steps. Another valuable property is that the spark timing variability does not change for different reference values: this guarantees a consistent engine behaviour throughout all possible operating conditions.

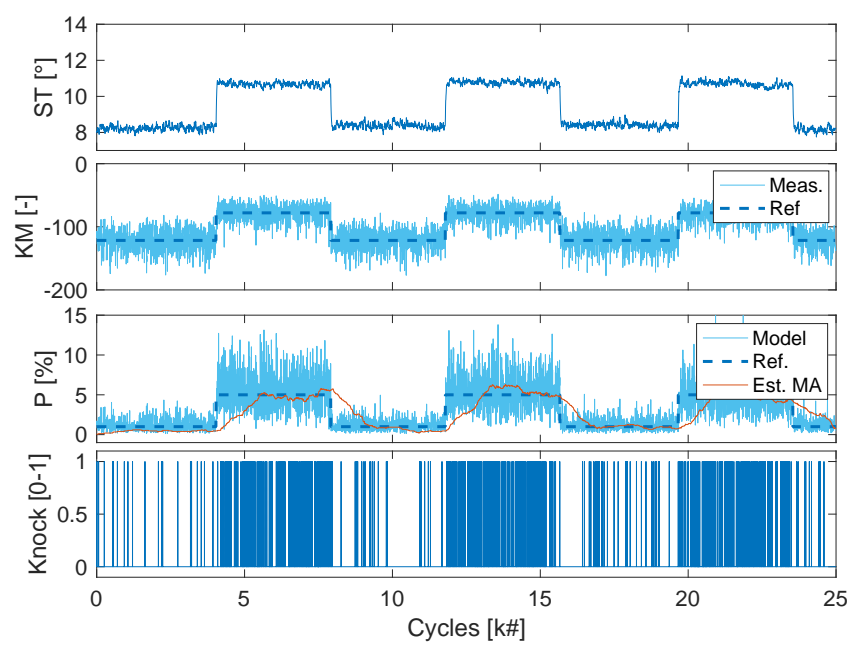

Fig. 6. Example of closed-loop knock margin control.

In order to check the effectiveness and the feasibility of the model-based tuning approach, Figure 7 shows the comparison between the closed-loop measured knock margin and the designed response; each plot of the comparison shows a different controller tuning. The consistency between expected and measured results is clear and shows the reliability of the model-based tuning approach. In Table II a summary of the

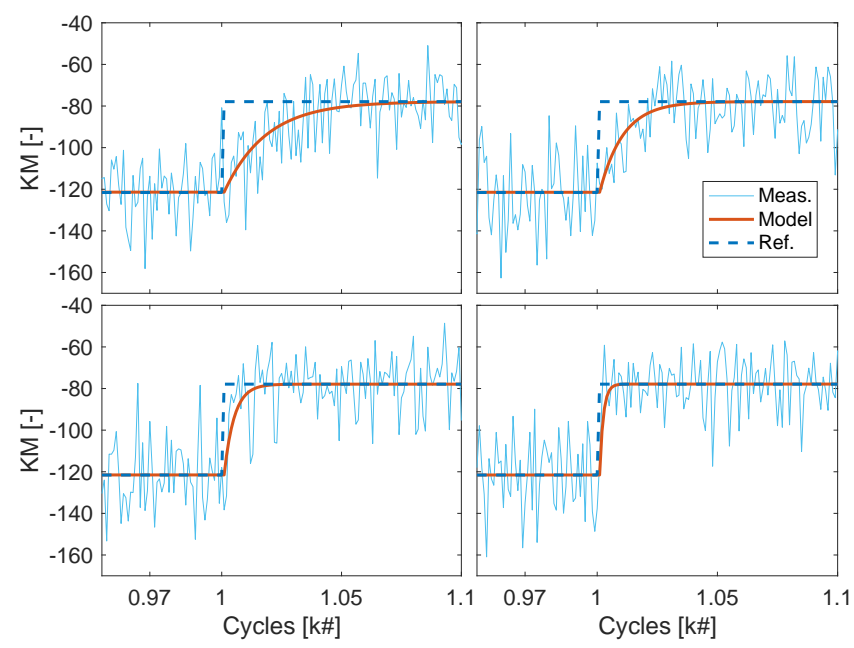

Fig. 7. Comparison between measured and designed closed-loop performance of KM controls.

main closed-loop properties is reported: for each controller, 
designed convergence time and the corresponding model reference poles are proposed in the first two columns. The third column reports the identified closed-loop system behaviour: the comparison with the designed closed-loop pole values quantitatively state the results of Figure 7. Finally, in the last column, the standard deviation of the ST recalls the discussed trade-off between speed of convergence and ST variability.

TABLE II

KNOCK MARGIN PI CONTROLLERS: MODEL REFERENCE REQUIRED SETTLING CYCLES TIME $t_{c y c}$, REQUESTED MODEL REFERENCE CLOSED-LOOP POLES $p$, EXPERIMENTALLY IDENTIFIED CLOSED-LOOP POLES $p_{i d}$ ON SYSTEM RESPONSE AND STANDARD DEVIATION ON CONTROL VARIABLE ST.

\begin{tabular}{ccccc} 
& $t_{c y c}$ & $p$ & $p_{i d}$ & ST std $\left[^{\circ}\right]$ \\
\hline PI 1 & 100 & 0.95 & 0.96 & 0.12 \\
PI 2 & 50 & 0.90 & 0.91 & 0.18 \\
PI 3 & 25 & 0.80 & 0.79 & 0.28 \\
PI 4 & 10 & 0.50 & 0.52 & 0.51
\end{tabular}

\section{B. Conventional controller comparison}

The same probability stepwise signal has been used as reference for the event-based, state-of-art knock control strategy. The overall performance of the controller are presented in Figure 8 where axis scales are kept equal to those of Figure 6 in order to ease a visual comparison. Of course, no knock margin plot is provided for this controller, since such variable is not anyhow evaluated in this strategy (as discussed in section III). The conventional knock controller features

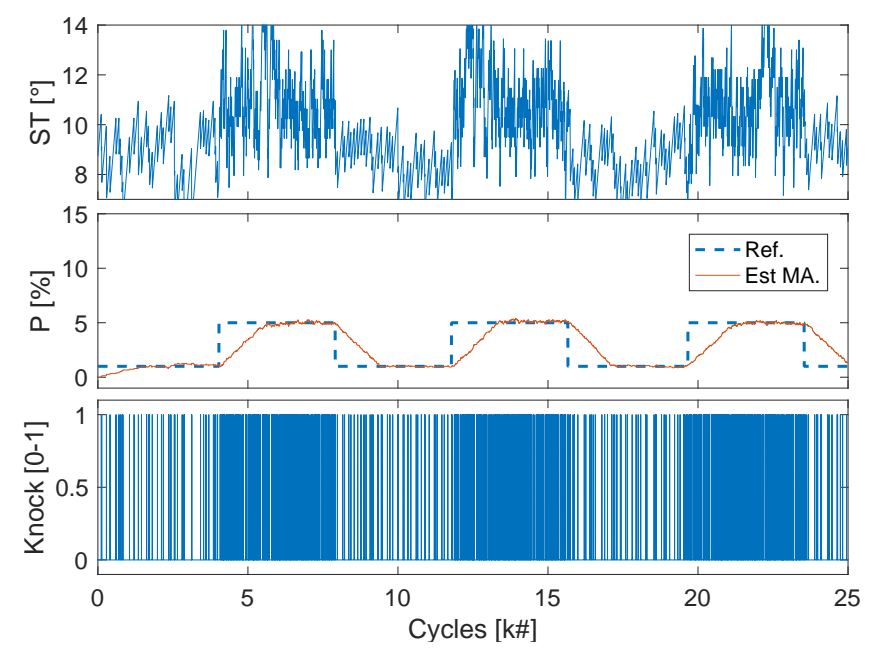

Fig. 8. Example of closed-loop conventional knock control.

an optimal tracking of the reference probability; however this happens at a cost, which can be seen by inspecting the control variable in the upper plot. In fact, the ST presents a greater variability, if compared with those of the knock margin control. Moreover, recalling equation (3), it should be noted how controller parameters are not constant but change with the reference probability: as a result, the behaviour of the spark timing change according to the operating conditions which is not a desirable engine property. In particular, when the reference probability is high, the controller acts more sharply and indeed this results in higher ST variance than the low probability case. In general, the ST variabiliy of the conventional controller is higher than any of the proposed KM controller tunings. In Figure 9, the ST distribution of all four KM controllers, in grayscale, is compared with the ST distribution of the conventional controller, in red.

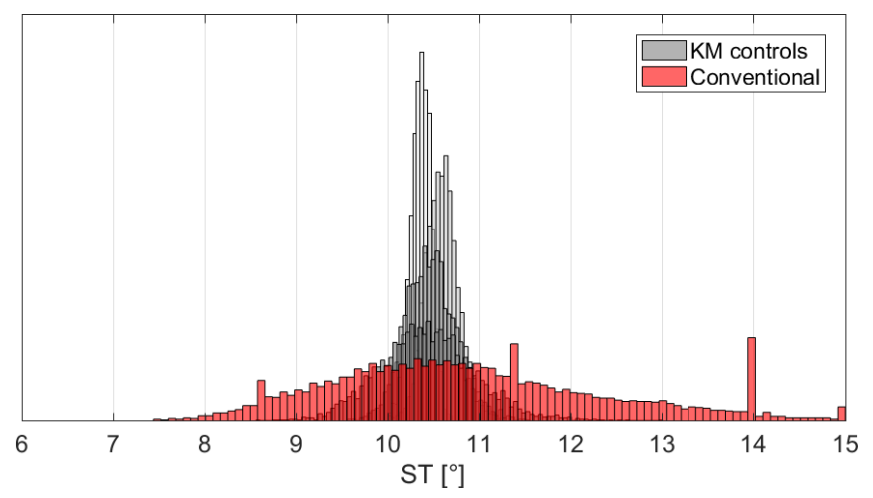

Fig. 9. Comparison between knock margin controllers and conventional one during a reference step decrease.

Another interesting comparison between conventional and $\mathrm{KM}$ control can be done by inspecting the steady-state settling time in response to the step change of reference probability. In Figure 10 this comparison is shown for an increasing probability step; the light grey lines represent the KM controllers (each for one of the different tuning considered) whereas the heavier red line shows the ST of the conventional controller. The dashed-dotted line indicates the moment when the reference changes. Conventional controller

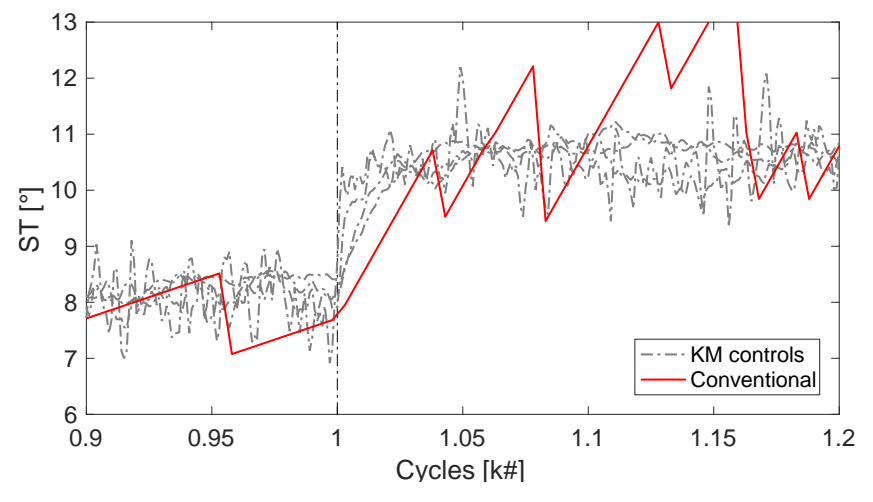

Fig. 10. Comparison between the knock margin controllers and the conventional one during a reference step increase.

response proves slower than the slowest KM controller, despite its parameters are tuned such that a higher $p_{\text {ref }}$ corresponds to a higher speed of the controller (see (3)). It should be remarked that the conventional controller has an additional degree of freedom in its tuning that would allow a further increase of the speed $\left(K_{r e t}\right)$ : however, as previously discussed, such a choice would lead to an increase 
of ST variance (which is already higher than those of KM controllers).

The comparison for the step-down scenario is provided in Figure 11, where in the upper and lower plots are shown two different steps. With this comparison it is possible to highlight the non-deterministic behaviour of the conventional controller. In fact, the ST reduction law of the conventional controller depends on the detection of a knock event (which is a non deterministic phenomenon, as already discussed): thus the steady-state settling time cannot be precisely-evaluated. This makes the overall engine behaviour non-constant, which is not a desirable feature. Opposite, the consistency of the KM control performance can be appreciated.

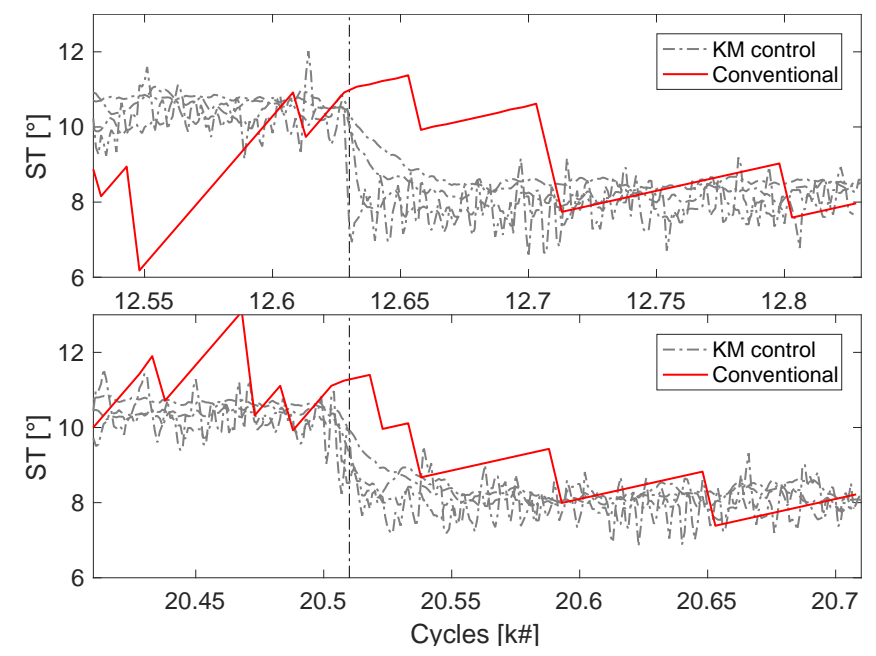

Fig. 11. Comparison between knock margin controllers and conventional one during two different reference step decrease.

\section{CONCLUSIONS AND FUTURE WORKS}

The knock phenomenon is highly stochastic and this complicates the process of fitting the problem of controlling it in a standard model-based design framework. Here, this is achieved by exploiting an advanced black-box modelling approach which provides an estimator of the knock margin and the knock probability. The knock margin is closed-loop regulated via a PI controller acting on the spark timing, while the probability model is inverted to compute a margin reference, given a reference probability. Results obtained on a experimental test bench show the effectiveness of the proposed control architecture. It allows a better tradeoff between controller speed and spark timing variability, compared to a conventional control. Differently from the conventional strategy, the proposed one shows consistent performance for all the considered values of the knock probability reference. However, for a fair comparison, it should be remarked that the conventional controller could outperform the KM one in case of evident knock margin modelling error or in case of its changes during the engine lifetime. In this case the reference probability would be transformed into a wrong knock margin target. To overcome this issue, the authors are currently working on an adaptive strategy, flanking the discussed KM control and capable of compensating slow-varying modelling errors and changes.

\section{REFERENCES}

[1] L. Guzzella and C. Onder, Introduction to Modeling and Control of Internal Combustion Engine Systems, 2nd ed. Springer, 2010.

[2] K. Kaji, S. Matsushige, M. Kanamaru, J. Takahashi, and S. Asano, "Development of knock sensor," in SAE Technical Paper. SAE International, October 1986.

[3] S. M. Dues, J. M. Adams, and G. A. Shinkle, "Combustion knock sensing: Sensor selection and application issues," in SAE Technical Paper. SAE International, February 1990.

[4] S.-H. Jang, Y.-G. Lee, T.-Y. Oh, and K.-S. Park, "An experimental study on knock sensing for a spark ignition engine," in SAE Technical Paper. SAE International, November 1993.

[5] E. Pipitone and L. D'Acquisto, "Development of a low-cost piezo film-based knock sensor," Proceedings of the Institution of Mechanical Engineers, Part D: Journal of Automobile Engineering, vol. 217, no. 8, pp. 691-699, 2003.

[6] U. Kiencke and L. Nielsen, Automotive control systems: for engine, driveline, and vehicle. Springer Science \& Business Media, 2005.

[7] J. C. P. Jones, K. R. Muske, J. Frey, and D. Scholl, "A stochastic knock control algorithm," in SAE Technical Paper. SAE International, April 2009.

[8] J. C. P. Jones, J. Frey, and K. R. Muske, "A fast-acting stochastic approach to knock control," IFAC Proceedings Volumes, vol. 42, no. 26, pp. $16-23,2009$.

[9] J. C. P. Jones, J. M. Spelina, and J. Frey, "Likelihood-based control of engine knock," IEEE Transactions on Control Systems Technology, vol. 21, no. 6, pp. 2169-2180, November 2013.

[10] A. Thomasson, H. Shi, T. Lindell, L. Eriksson, T. Shen, and J. C. P. Jones, "Experimental validation of a likelihood-based stochastic knock controller," IEEE Transactions on Control Systems Technology, vol. 24, no. 4, pp. 1407-1418, July 2016.

[11] U. Lezius, M. Schultalbers, W. Drewelow, and B. Lampe, "Improvements in knock control," in Mediterranean Conference on Control \& Automation, 2007. MED'07. IEEE, June 2007, pp. 1-5.

[12] M. Penese, C. F. Damasceno, A. Bucci, and G. Montanari, "Sigma ${ }^{\circledR}$ on knock phenomenon control of flexfuel engines," in SAE Technical Paper. SAE International, November 2005, p. 3990.

[13] P. Bares, D. Selmanaj, C. Guardiola, and C. Onder, "Knock probability estimation through an in-cylinder temperature model with exogenous noise," Mechanical Systems and Signal Processing, vol. 98, pp. 756769, 2018.

[14] G. Panzani, C. Onder, and F. Ostman, "Engine knock margin estimation using in-cylinder pressure measurements," IEEE/ASME Transactions on Mechatronics, 2016.

[15] J. M. Spelina, J. C. P. Jones, and J. Frey, "Stochastic simulation and analysis of a classical knock controller," International Journal of Engine Research, vol. 16, no. 3, pp. 461-473, 2015. 